ternational Electronic Journal of Algebra

Volume 28 (2020) 141-155

DOI: $10.24330 /$ ieja.768206

\title{
DIFFERENT TYPES OF $G$-PRIME IDEALS ASSOCIATED TO A GRADED MODULE AND GRADED PRIMARY DECOMPOSITION IN A GRADED PRÜFER DOMAIN
}

\author{
Ajim Uddin Ansari, B. K. Sharma and Shiv Datt Kumar \\ Received: 8 October 2019; Revised: 12 January 2020; Accepted: 20 January 2020 \\ Communicated by A. Çiğdem Özcan
}

\begin{abstract}
In this paper, we introduce the notion of graded Prüfer domain as a generalization of Prüfer domain to the graded case. We generalize several types of prime ideals associated to a module over a ring to the graded case and prove that most of them coincide over a graded Prüfer domain. Moreover, we investigate the graded primary decomposition of graded ideals in a graded Prüfer domain under certain conditions and give some applications of it.
\end{abstract}

Mathematics Subject Classification (2020): 16W50, 13A02, 13A15, 13 F05

Keywords: Graded ring, graded module, graded Prüfer domain, graded primary decomposition

\section{Introduction}

The theory of rings which are graded by a finitely generated abelian group came in light mainly when homogeneous coordinate rings for toric varieties were introduced in algebraic geometry [5]. The theory of graded rings and modules can be considered as an extension of the ring and module theory which has been studied by many authors (See [1], [2], [3], [5], [10], [11], [13], [14] and [15]). A comparison between global primary decomposition of coherent sheaves over a toric variety and graded primary decomposition of graded ideals is given in [15]. Graded primary decomposition over a $G$-graded Noetherian ring has been discussed in [4] for the case when $G$ is a finitely generated torsion free abelian group. M. Perling and S.D. Kumar [14] used this concept to extend the graded primary decomposition over a $G$-graded Noetherian ring for the case when $G$ is an arbitrary finitely generated abelian group. In [11], S. Behara and S.D. Kumar investigated the uniqueness of graded primary decomposition in graded modules. It is well known that if a ring is graded Noetherian, then the graded primary decomposition exists but in graded non-Noetherian case, it exists rarely. In [8], L. Fuchs and E. Mosteig gave a characterization of primary decomposition of ideals in a Prüfer domain (not necessarily 
Noetherian). Further, the theory of different types of prime ideals associated to a module over a commutative ring is a very important concept in commutative algebra. Several authors have studied such types of prime ideals (see [4], [6], [7] and [9]). In [9], J. Iroz and D.E. Rush discussed many important properties about these types of prime ideals. In [6], P. Dutton introduced a quite different notion of associated prime ideal called attached prime ideal. Recently in [7], N. Epstein and J. Shapiro exhibited why strong Krull prime ideals can be considered as the best non-Noetherian generalization of associated prime ideals by proving many important results with the help of strong Krull prime ideals which were enjoyed by an associated prime ideal in Noetherian case. To study the theory of graded rings and modules in more detail, one needs the graded analogues of different notions of prime ideals associated to a module. Although there has been done a lot of work in the area of graded rings and modules but a generalization of different notions of prime ideals associated to a module and primary decomposition in a Prüfer domain to the graded case is not found anywhere. This paper tries to fill this gap.

This paper discusses two objectives. Section 3 discusses the first objective of the paper which is devoted to the study of different types of $G$-prime ideals associated to a $G$-graded module where $G$ is an arbitrary finitely generated abelian group. We generalize different notions of prime ideals associated to a module (which is discussed in [9]) to the graded case and make use of some examples to show the difference between graded and non-graded cases. We show by Example 3.7 and Example 3.14 that the graded versions of all the notions of prime ideals associated to a module are different from the non-graded versions. It is well known that over a Noetherian ring all the notions of associated prime ideals defined in [9] coincide. For the graded case, a natural question arises, for which graded non-Noetherian ring most of the notions of $G$-prime ideals associated to a graded module coincide. To answer this question, first we introduce the notion of graded Prüfer domain as a generalization of Prüfer domain to the graded case, then we show that most of the notions of $G$-prime ideals associated to a graded module over a graded Prüfer domain under certain conditions coincide (Theorem 3.9 and Proposition 3.12). Graded valuation domains and graded Prüfer domains were studied in [1] and [2], where the authors assumed that the graded domains are integral domains. So our definitions of graded valuation domain and graded Prüfer domain are generalizations of [1] and [2]. We also prove the graded local-global principle of a graded module in terms of strong $G$-Krull prime ideals (Theorem 3.18). 
Section 4 discusses the second objective of the paper which is devoted to the study of graded primary decomposition of a graded ideal in a graded Prüfer domain. For an arbitrary finitely generated abelian group $G$, we establish a $G$-graded primary decomposition of a graded ideal in a graded Prüfer domain under certain conditions (Theorem 4.2). H. Khashan [10] introduced the notion of QGR ring. As an application of Theorem 4.2, we show that a graded Prüfer domain under certain conditions is a QGR ring (Proposition 4.6). Some applications of Theorem 4.2 are also discussed.

\section{Preliminaries}

Throughout this paper, $G$ is a finitely generated abelian group and all rings are assumed to be commutative rings with identity unless otherwise stated.

Let $A$ be a $G$-graded ring and $M$ be a $G$-graded $A$-module. We denote by $h(A), \operatorname{Spec}^{G}(A), \operatorname{Max}^{G}(A)$, the set of all homogeneous elements, $G$-prime ideals, $G$-maximal ideals of $A$ respectively. The set of all homogeneous elements of $M$ is denoted by $h(M)$. $A$ is called a $G$-graded integral domain if for $a, b \in h(A), a b=0$ implies that either $a=0$ or $b=0$. Note that a $G$-graded integral domain need not be an integral domain. A $G$-graded integral domain is called a $G$-graded field if each nonzero homogeneous element has a multiplicative inverse. $A$ is called a $G$-graded Noetherian ring if it satisfies the ACC on graded ideals of $A$. Let $S$ be a multiplicatively closed subset of $h(A)$ containing $1_{A}$. Then the ring of fraction $S^{-1} A$ is a graded ring called a $G$-graded ring of fraction. Indeed $S^{-1} A=\oplus_{g \in G}\left(S^{-1} A\right)_{g}$, where $\left(S^{-1} A\right)_{g}=\left\{\frac{a}{s}: a \in h(A), s \in S\right.$ and $g=(\text { degs })^{-1}($ dega $\left.)\right\}$. Let $P$ be a $G$ prime ideal of $A$ and $S=h(A) \backslash P$. We denote $S^{-1} A$ by $A_{P}^{G}$ and call it the $G$-graded localization of $A$ at $P$. This ring $A_{P}^{G}$ has a unique $G$-maximal ideal $S^{-1} P$ denoted by $P A_{P}^{G}$. Also, $S^{-1} M$ is denoted by $M_{P}^{G}$ called the $G$-graded localization of $M$ at $P$. We denote by $h\left(M_{P}^{G}\right), h\left(A_{P}^{G}\right), h\left(P A_{P}^{G}\right), h\left(I A_{P}^{G}\right)$, the set of all homogeneous elements of $M_{P}^{G}, A_{P}^{G}, P A_{P}^{G}, I A_{P}^{G}$ respectively where $I$ is a graded ideal of $A$. The graded support of $M$ is defined as $\operatorname{Supp}_{A}^{G}(M)=\left\{P \in \operatorname{Spec}^{G}(A): M_{P}^{G} \neq 0\right\}$. The graded radical of a graded ideal $I$ is denoted by $\operatorname{Gr}(I)$ and defined as $\operatorname{Gr}(I)=\{a=$ $\Sigma_{g \in G} a_{g} \in A$ : for all $g \in G$, there exists an integer $n_{g} \geq 1$ such that $\left.a_{g}^{n_{g}} \in I\right\}$. A graded ideal $Q$ of $A$ is said to be a $G$-primary ideal if $Q \neq A$ and if $a, b \in h(A)$ such that $a b \in Q$, then $a \in Q$ or $b \in \operatorname{Gr}(Q)$. For a $G$-primary ideal $Q, \operatorname{Gr}(Q)=P$ where $P$ is a $G$-prime ideal and we call $Q$ a $G$-graded $P$-primary ideal. A decomposition of a graded ideal $I$ of the type $Q_{1} \cap Q_{2} \cap \cdots \cap Q_{r}$ where each $Q_{i}$ is a $G$-graded 
$P_{i}$-primary ideal of $A$ is said to be a $G$-graded primary decomposition of $I$ in $A$ and it is said to be reduced if $Q_{i} \nsupseteq \bigcap_{j \neq i} Q_{j}$ and each $P_{i}$ are distinct.

For more details of graded rings and graded modules [10], [13], [14] and [16] are referred.

Definition 2.1. [10] The gr-dimension of a $G$-graded ring $A$ is defined to be the supremum of lengths of all chains of distinct $G$-prime ideals of $A$ and is denoted by $\operatorname{gr}-\operatorname{dim}(A)$.

It is clear from the definition that if $A$ is a $G$-graded integral domain such that $\operatorname{gr}-\operatorname{dim}(A)=1$, then a nonzero $G$-prime ideal of $A$ is a $G$-maximal ideal.

Definition 2.2. [10] A $G$-graded ring $A$ is called a QGR-ring if any graded ideal of $A$ can be written as a product of finitely many $G$-primary ideals of $A$.

Definition 2.3. A $G$-graded integral domain $A$ is said to be of $G$-finite character if any nonzero non-unit homogeneous element of $A$ is contained in only finitely many $G$-maximal ideals of $A$.

Proposition 2.4. [10] Let $I$ and $J$ be graded ideals in a G-graded ring $A$. Then $(I: J)=\{a \in A: a J \subseteq I\}$ is a graded ideal of $A$.

Proposition 2.5. [16] Let $Q$ be a graded ideal in a G-graded ring $A$ such that $\operatorname{Gr}(Q)=m$, a $G$-maximal ideal. Then $Q$ is a G-graded m-primary ideal.

Definition 2.6. [12] Let $A$ be an integral domain. Then

(1) $A$ is called a valuation domain if for all $a, b$ in $A$ either $a$ divides $b$ or $b$ divides $a$.

(2) $A$ is called a Prüfer domain if its localization $A_{P}$ is a valuation domain for each prime ideal $P$ of $A$.

Theorem 2.7. [8] Let $A$ be a Prüfer domain. Then any nonzero ideal of $A$ can be written as an intersection of finitely many primary ideals if and only if $A$ is of finite character and of Krull dimension 1. Further, any ideal can be uniquely expressed as the product of primary ideals.

\section{Graded Prüfer domains and properties of $G$-prime ideals associated to a graded module}

Let $G$ be a finitely generated abelian group. We define a $G$-graded Prüfer domain as a generalization of a Prüfer domain to the graded case. 
Definition 3.1. Let $A$ be a $G$-graded integral domain. Then

(1) $A$ is called a $G$-graded valuation domain if for any $a, b \in h(A)$ either $a$ divides $b$ or $b$ divides $a$.

(2) $A$ is called a $G$-graded Prüfer domain if its graded localization $A_{P}^{G}$ is a $G$-graded valuation domain for each $G$-prime ideal $P$ of $A$.

Proposition 3.2. Every finitely generated graded ideal in a G-graded valuation domain is principal.

Proof. Let $A$ be a $G$-graded valuation domain. Let $\left\{a_{1}, a_{2}, \ldots, a_{n}\right\}$ be a set of homogeneous generators of a graded ideal $I$ of $A$. Then there exists $i$ such that $a_{i}$ divides $a_{j}$ for all $j$. Consequently, $I$ is a principal ideal generated by $a_{i}$.

Proposition 3.3. Let $A$ be a G-graded valuation domain and I be its graded ideal. Then $G r(I)$ is a G-prime ideal.

Proof. Let $a, b \in h(A)$ such that $a b \in G r(I)$. Then $(a b)^{n} \in I$ for some integer $n \geq 1$. Suppose $a$ divides $b$. Then $b^{2 n} \in I$, and so $b \in \operatorname{Gr}(I)$. Similarly if $b$ divides $a$, then $a \in \operatorname{Gr}(I)$. Thus $\operatorname{Gr}(I)$ is a $G$-prime ideal.

Example 3.4. Every Prüfer domain is trivially a $G$-graded Prüfer domain.

We now give an example to show that a $G$-graded Prüfer domain need not be a Prüfer domain.

Example 3.5. Let $A=F[x]$, the polynomial ring over a field $F$ and let $G=$ $\mathbb{Z}_{2}=\{\overline{0}, \overline{1}\}$. Then $A$ is a $G$-graded ring with $A_{\overline{0}}=F+F x^{2}+F x^{4}+\cdots$ and $A_{\overline{1}}=F x+F x^{3}+F x^{5}+\cdots$. Consider the quotient ring $R=A / I$, where $I=$ $\left(x^{2}-1\right)$, a graded ideal generated by a homogeneous element $x^{2}-1$ of $A$. Consider the induced grading on $R$ given by $R=R_{\overline{0}} \oplus R_{\overline{1}}$, where $R_{\overline{0}}=\left(A_{\overline{0}}+I\right) / I$ and $R_{\overline{1}}=\left(A_{\overline{1}}+I\right) / I$. Then $R$ is a $G$-graded field since $I$ is a $G$-maximal ideal of $A$, and so $R$ is a $G$-graded Prüfer domain but not a Prüfer domain since it is not an integral domain.

Now we generalize several types of prime ideals associated to a module over a ring to the graded case. A non-graded version of these definitions can be found in $[9]$.

Definition 3.6. Let $A$ be a $G$-graded ring and $M$ be a $G$-graded $A$-module. Suppose $P$ is a $G$-prime ideal of $A$. Then 
(1) $P$ is a $G$-associated prime ideal to $M$ if $P=A n n(x)$ for some $0 \neq x \in h(M)$ where $\operatorname{Ann}(x)$ denotes the annihilator of $x$. The set of these $G$-prime ideals is denoted by $A s s_{A}^{G}(M)$. They are discussed in [14].

(2) $P$ is a strong $G$-Krull prime ideal to $M$ if for every finitely generated graded ideal $I$ of $A$ such that $I \subseteq P$, there exists $0 \neq x \in h(M)$ such that $I \subseteq$ $\operatorname{Ann}(x) \subseteq P$. The set of all strong $G$-Krull prime ideals is denoted by $S K_{A}^{G}(M)$. They are discussed in [3].

(3) $P$ is a weak $G$-Bourbaki prime ideal to $M$ if there exists $0 \neq x \in h(M)$ such that $P$ is minimal over $\operatorname{Ann}(x)$. These $G$-prime ideals will be denoted by $A s s_{f}^{G}(M)$.

(4) $P$ is a $G$-Krull prime ideal to $M$ if for every homogeneous element $a \in P$, there exists $0 \neq x \in h(M)$ such that $a \in \operatorname{Ann}(x) \subseteq P$. The set of all $G$-Krull prime ideals is denoted by $K_{A}^{G}(M)$.

(5) $P$ is a $G$-Zariski-Samuel prime ideal to $M$ if there exists $0 \neq x \in h(M)$ such that $\operatorname{Ann}(x)$ is a $G$-graded $P$-primary ideal of $A$. The set of all $G$ Zariski-Samuel prime ideals to $M$ is denoted by $Z S_{A}^{G}(M)$.

Like the ungraded case one can prove that $A s s_{A}^{G}(M) \subseteq Z S_{A}^{G}(M) \subseteq A s s_{f}^{G}(M) \subseteq$ $S K_{A}^{G}(M) \subseteq K_{A}^{G}(M)$ (see [9]). We now show by the following example that a $G$ associated, $G$-Zariski-Samuel, $G$-Krull, strong $G$-Krull and weak $G$-Bourbaki prime ideals to $M$ need not be associated, Zariski-Samuel, Krull, strong Krull and weak Bourbaki ideals to $M$ respectively.

Example 3.7. Let $A=\mathbb{Q}[x]$ and $M=A / I$, where $I$ is the ideal generated by $x^{2}-1$ in $A$. Consider the group $G=\mathbb{Z}_{2}=\{\overline{0}, \overline{1}\}$ and grading $A=A_{\overline{0}} \oplus A_{\overline{1}}$, $M=M_{\overline{0}} \oplus M_{\overline{1}}$, where $A_{\overline{0}}=\mathbb{Q}+\mathbb{Q} x^{2}+\mathbb{Q} x^{4}+\cdots, A_{\overline{1}}=\mathbb{Q} x+\mathbb{Q} x^{3}+\mathbb{Q} x^{5}+\cdots$, $M_{\overline{0}}=(A / I)_{\overline{0}}=\left\{f+I: f \in A_{\overline{0}}\right\}$ and $M_{\overline{1}}=(A / I)_{\overline{1}}=\left\{f+I: f \in A_{\overline{1}}\right\}$. Then $M$ is a $G$-graded $A$-module. Consider the $G$-prime ideal $P=\left(x^{2}-1\right)$ which is clearly not a prime ideal of $A$. Clearly $P=A n n(1+I)$ where $1+I \in h(M)$. Thus $P$ is a $G$-associated prime ideal to $M$. Hence $P$ is a $G$-Zariski-Samuel, $G$-Krull, strong $G$-Krull and weak $G$-Bourbaki prime ideals to $M$, but it is not a Zariski-Samuel, Krull, strong Krull and weak Bourbaki prime ideals to $M$ respectively since $P$ is not a prime ideal of $A$.

The next example shows that a $G$-Krull prime ideal need not be a strong $G$-Krull prime ideal.

Example 3.8. Let $A=K\left[x_{1}, x_{2}, x_{3}, \ldots, x_{n}, \ldots\right]$, the polynomial ring in infinitely many indeterminates over a field $K$. Consider the ideal $I=\left(x_{1}^{m}, x_{2}^{m}, x_{3}^{m}, \ldots\right)$ of 
$A$ where $m \geq 2$. Let $M=A / I$ be an $A$-module trivially graded by $G$. Consider the prime ideal $P=\left(x_{1}, x_{2}, \ldots, x_{n}\right)$ of $A$. Since $x_{i} \in A n n\left(x_{i}^{m-1}+I\right) \subseteq P$ and $x_{i}^{m-1}+I$ is a nonzero element of $M$, then for all $a \in P$, there exists a nonzero $m \in M$ such that $a \in A n n(m) \subseteq P$. Thus $P$ is a $G$-Krull prime ideal to $M$. If $P \in$ $S K_{A}^{G}(M)$, then $P=A n n(f)$ for some nonzero $f \in M$ since $P$ is finitely generated. Consequently, $x_{i}^{m} \in P=A n n(f)$ for all $i$. This implies $x_{i} \in P=A n n(f)$ for all $i$, which is a contradiction. Hence $P$ is not a strong $G$-Krull prime ideal to $M$.

Now one can ask when both the sets $S K_{A}^{G}(M)$ and $K_{A}^{G}(M)$ will be the same. The following theorem gives an affirmative answer to this question.

Theorem 3.9. Let $A$ be a $G$-graded Prüfer domain and $M$ be a $G$-graded A-module. Then $S K_{A}^{G}(M)=K_{A}^{G}(M)$.

Proof. Let $P \in K_{A}^{G}(M)$. Let $0 \neq \frac{b}{t} \in h\left(P A_{P}^{G}\right)$ where $b$ is a homogeneous element of $P$ and $t \in h(A) \backslash P$. Then there is a $0 \neq x \in h(M)$ such that $b \in A n n(x) \subseteq P$. Consequently, $\frac{b}{t} \in \operatorname{Ann}\left(\frac{x}{1}\right) \subseteq P A_{P}^{G}$ and hence $P A_{P}^{G} \in K_{A_{P}^{G}}^{G}\left(M_{P}^{G}\right)$. Now, let $L$ be a finitely generated graded ideal of $A_{P}^{G}$ such that $L \subseteq P A_{P}^{G}$. Since $A_{P}^{G}$ is a $G$ graded valuation domain, then by Proposition 3.2, $L$ is a principal ideal generated by a homogeneous element $\frac{a}{t}$ say. Hence there exists a $0 \neq \frac{y}{s} \in h\left(M_{P}^{G}\right)$ such that $L \subseteq A n n\left(\frac{y}{s}\right) \subseteq P A_{P}^{G}$ and so $P A_{P}^{G} \in S K_{A_{P}^{G}}^{G}\left(M_{P}^{G}\right)$.

Now, let $I$ be a finitely generated graded ideal of $A$ such that $I \subseteq P$. Then $I A_{P}^{G}$ is a finitely generated graded ideal of $A_{P}^{G}$ such that $I A_{P}^{G} \subseteq P A_{P}^{G}$. This implies that there exists a $0 \neq \frac{y}{t} \in h\left(M_{P}^{G}\right)$ such that $I A_{P}^{G} \subseteq A n n\left(\frac{y}{t}\right) \subseteq P A_{P}^{G}$. Let $a \in I$. Then $\frac{a}{1} \in I A_{P}^{G}$, and so $\frac{a y}{t}=0$. This implies that there exists $s \in h(A) \backslash P$ such that $a \in A n n(s y)$ since $I$ is finitely generated. Clearly, $s y \neq 0$ since $\frac{y}{t} \neq 0$. Now $r \in A n n(s y)$ implies that $r s \in P$ since $\frac{y}{t} \neq 0$, and so $r \in P$. Thus $I \subseteq A n n(s y) \subseteq P$ where $0 \neq s y \in h(M)$. Hence $P \in S K_{A}^{G}(M)$.

It was shown in [7] that a Krull prime is not a proper generalization of an associated prime in the sense that there is a module $M$ over a Noetherian ring $A$ such that $A s s_{A}(M) \neq K_{A}(M)$ where $A s s_{A}(M)$ and $K_{A}(M)$ denotes the set of all associated prime ideals and Krull prime ideals to $M$ respectively, and so it also happens in the graded case. Now one can ask for which $G$-graded Noetherian ring $A, A s s_{A}^{G}(M)=K_{A}^{G}(M)$. We have the following corollary.

Corollary 3.10. Let $A$ be a G-graded Prüfer domain and $M$ be a G-graded $A$ module. If $A$ is a $G$-graded Noetherian ring, then $\operatorname{Ass}_{A}^{G}(M)=K_{A}^{G}(M)$. 
Proof. Let $P \in S K_{A}^{G}(M)$. Then there exists a $0 \neq x \in h(M)$ such that $P \subseteq$ $\operatorname{Ann}(x) \subseteq P$, i.e., $P=A n n(x)$ since $P$ is finitely generated. Consequently, $A s s_{A}^{G}(M)=S K_{A}^{G}(M)$. Also by Theorem 3.9, we have $S K_{A}^{G}(M)=K_{A}^{G}(M)$. Hence $A s s_{A}^{G}(M)=K_{A}^{G}(M)$.

For a $G$-graded $A$-module $M, Z S_{A}^{G}(M) \subseteq S K_{A}^{G}(M)$ but the converse need not be true even if $A$ is a $G$-graded Prüfer domain. Consider the following example.

Example 3.11. Let $A=\mathbb{Z}+x \mathbb{Q}[x]$ and $G=\mathbb{Z}$. Consider the grading on $A$, $A=\bigoplus_{n \in \mathbb{Z}} A_{n}$, where $A_{0}=\mathbb{Z}, A_{n}=\mathbb{Q} x^{n}$ if $n \geq 1$ and $A_{n}=0$ if $n<0$. Then $A$ is a $G$-graded Prüfer domain. Let $I=(x)=\left\{a_{0} x+a_{1} x^{2}+\cdots+a_{n} x^{n+1}: a_{0} \in \mathbb{Z}, a_{i} \in\right.$ $\mathbb{Q}, n \geq 0\}$. Then $I$ is a graded ideal of $A$. Consider $M=A / I$ as an $A$-module. Consider the grading on $M, M=\bigoplus_{n \in \mathbb{Z}} M_{n}$, where $M_{n}=(A / I)_{n}=\left\{e+I: e \in A_{n}\right\}$ if $n \geq 0$ and $M_{n}=0$ if $n<0$. Then $M$ is a $G$-graded $A$-module. Let $P=x \mathbb{Q}[x]$. Then $P$ is a $G$-prime ideal of $A$ such that $I \subseteq P$. We show that $P \in S K_{A}^{G}(M)$ but $P \notin Z S_{A}^{G}(M)$.

Let $f \in P$ be a homogeneous element. Then $f=a x^{i}$ for some integer $i \geq 1$ where $a \in \mathbb{Q}$. Write $a=\frac{p}{q}$ for some $p, q \in \mathbb{Z}$ with $q \neq 0$. Then $f \in A n n(q+I)$ where $q+I \in h(M) \backslash\{0\}$. Also, let $g \in \operatorname{Ann}(q+I)$ be a homogeneous element. Then $q g \in I \subseteq P$. This implies that $g \in P$ since $q \notin P$. Thus for each homogeneous element $f \in P$, there exists a nonzero $q+I \in h(M)$ such that $f \in A n n(q+I) \subseteq P$, and so $P \in K_{A}^{G}(M)$. By Theorem $3.9, P \in S K_{A}^{G}(M)$. On contrary suppose $P$ is a $G$-Zariski-Samuel prime ideal of $A$. Then there exists a nonzero $h+I \in h(M)$ such that $A n n(h+I)$ is a $G$-graded $P$-primary ideal of $A$, and so $G r(A n n(h+I))=P$. Now, $h \notin I$ implies that $h+I=\alpha+I$ or $h+I=\beta x+I$ for some $\alpha \in \mathbb{Z}$ and $\beta \in \mathbb{Q} \backslash \mathbb{Z}$. Assume $h+I=\alpha+I$. Write $x=d \frac{x}{d}$ where $d \in \mathbb{Z}$ such that $d$ does not divide $\alpha$. Then $x \in A n n(h+I)$ but neither $\frac{x}{d}$ nor $d^{n}$ belongs to $A n n(h+I)$ for any integer $n \geq 1$, which is a contradiction. Assume $h+I=\beta x+I$. Write $\beta=\frac{r}{s}$ for some nonzero $r, s \in \mathbb{Z}$. Then $s \in A n n(h+I)$ but $s \notin P$, which is a contradiction since $\operatorname{Ann}(h+I) \subseteq P$. Hence $P \notin Z S_{A}^{G}(M)$.

However, we have the following.

Proposition 3.12. Let $A$ be a $G$-graded valuation domain with $g r-\operatorname{dim}(A) \leq 1$ and $M$ be a G-graded A-module. Then $Z S_{A}^{G}(M)=A s s_{f}^{G}(M)=S K_{A}^{G}(M)$.

Proof. It suffices to show $S K_{A}^{G}(M) \subseteq Z S_{A}^{G}(M)$. Let $P \in S K_{A}^{G}(M)$. Choose a finitely generated graded ideal $I$ of $A$ such that $I \subseteq P$. Then there exists a $0 \neq x \in h(M)$ such that $I \subseteq A n n(x) \subseteq P$. This implies that $G r(A n n(x)) \subseteq P$. By 
Proposition 3.3, $G r(\operatorname{Ann}(x))=Q$ where $Q$ is a $G$-prime ideal of $A$. But gr-dim $(A)$ $\leq 1$ implies that $Q=P$, a $G$-maximal ideal of $A$. Hence by Proposition 2.5, Ann $(x)$ is a $G$-graded $P$-primary ideal of $A$, and so $P \in Z S_{A}^{G}(M)$.

Corollary 3.13. Let $A$ be a G-graded Prüfer domain with gr-dim $(A) \leq 1$ and $M$ be a G-graded A-module. Then $Z S_{A_{P}^{G}}^{G}\left(M_{P}^{G}\right)=A s s_{f}^{G}\left(M_{P}^{G}\right)=S K_{A_{P}^{G}}^{G}\left(M_{P}^{G}\right)=\left\{P A_{P}^{G}\right\}$ for all $P \in \operatorname{Supp}_{A}^{G}(M)$.

We now show by an example that associated prime, Zariski-Samuel, Krull, strong Krull and weak Bourbaki prime ideals to $M$ need not be $G$-associated, $G$-ZariskiSamuel, $G$-Krull, strong $G$-Krull and weak $G$-Bourbaki prime ideals to $M$ respectively.

Example 3.14. Let $A=\mathbb{Q}[x]$ and $M=A / I$ where $I=\left(x^{2}-1\right)$ is an ideal in $A$. Consider the group $G=\mathbb{Z}_{2}$. Then $M$ is a $G$-graded $A$-module with grading as in Example 3.7. Consider the prime ideal $P=(x+1)$ of $A$. Then $P=\operatorname{Ann}((x-$ $1)+I$ ), and so $P$ is an associated prime ideal to $M$. Consequently, $P$ is a ZariskiSamuel, Krull, strong Krull and weak Bourbaki prime ideal to $M$ but none of the $G$-Zariski-Samuel, $G$-Krull, strong $G$-Krull and weak $G$-Bourbaki prime ideal to $M$ respectively since $P$ is not a $G$-prime ideal of $A$.

However we have the following.

Proposition 3.15. Let $M$ be a G-graded A-module. Let $P$ be a strong Krull prime ideal in non-graded case and $P^{\prime}$ be the largest graded ideal contained in $P$. Then $P^{\prime} \in S K_{A}^{G}(M)$.

Proof. Clearly $P^{\prime}$ is a $G$-prime ideal of $A$. Let $I$ be a finitely generated graded ideal of $A$ such that $I \subseteq P^{\prime}$. Then $I$ is a finitely generated ideal such that $I \subseteq P$, and so by definition of strong Krull prime ideal, there exists a $0 \neq x \in M$ such that $I \subseteq A n n(x) \subseteq P$. Write $x=x_{g_{1}}+x_{g_{2}}+\cdots+x_{g_{n}}$ where $0 \neq x_{g_{i}} \in h(M)$. Then $\prod_{i=1}^{n} A n n\left(x_{g_{i}}\right) \subseteq \bigcap_{i=1}^{n} A n n\left(x_{g_{i}}\right) \subseteq A n n(x) \subseteq P$. Then there exists $j$ such that $\operatorname{Ann}\left(x_{g_{j}}\right) \subseteq P$. Consequently, $I \subseteq A n n\left(x_{g_{j}}\right) \subseteq P^{\prime}$ since $A n n\left(x_{g_{j}}\right)$ is a graded ideal and all homogeneous elements of $P$ are in $P^{\prime}$. Hence $P^{\prime} \in S K_{A}^{G}(M)$.

Proposition 3.15 also holds if we replace $S K_{A}^{G}(M)$ by $A s s_{A}^{G}(M)$ or $K_{A}^{G}(M)$. Further, Example 3.7 shows that a $G$-Zariski-Samuel prime ideal need not be a Zariski-Samuel prime ideal. There are two reasons: the first one is that a $G$-prime ideal need not be a prime ideal (see Example 3.7) and the other is that a $G$-primary ideal need not be a primary ideal.

The following example shows that a $G$-primary ideal need not be a primary ideal. 
Example 3.16. Let $A=\mathbb{Q}[x]$ be the polynomial ring with $\mathbb{Z}_{2}$-grading as in Example 3.7. Then $Q=\left(x^{4}+x^{2}+1\right)$ is a $G$-graded primary ideal but not a primary ideal since $x^{4}+x^{2}+1 \in Q$ but $x^{2}+x+1 \notin Q$ and $\left(x^{2}-x+1\right)^{n} \notin Q$ for any integer $n \geq 1$.

Now we wish to know when a G-Zariski-Samuel prime ideal is a Zariski-Samuel prime ideal. We have the following theorem.

Theorem 3.17. Let $G$ be a finitely generated torsion free abelian group and $M$ be a G-graded A-module. Then every G-Zariski-Samuel prime ideal is a Zariski-Samuel prime ideal.

Proof. First we prove that a $G$-primary ideal of $A$ is a primary ideal. Let $Q$ be a $G$-primary ideal of $A$. On the contrary suppose $Q$ is not a primary ideal of $A$. Then there exists $a, b \in A$ such that $a b \in Q$ but $a \notin Q$ and $b \notin \operatorname{Rad}(Q)$ where $\operatorname{Rad}(Q)$ denotes the radical of $Q$. Then we can write $b=d+c$ where $d \in \operatorname{Rad}(Q)$ and $c=\sum_{h \in G} b_{h}$ with $b_{h} \notin \operatorname{Rad}(Q)$ for all $h \in G$. Since $G$ is torsion free, $G$ admits a total order $\leq$ compatible with the group structure, i.e., if $\alpha \leq \beta$ where $\alpha, \beta \in G$, then $\alpha+\gamma \leq \beta+\gamma$ for all $\gamma \in G$. We can write $a=a_{g_{1}}+a_{g_{2}}+\cdots+a_{g_{n}}$, $c=b_{h_{1}}+b_{h_{2}}+\cdots+b_{h_{m}}$ with $g_{1}<g_{2}<\cdots<g_{n}$ and $h_{1}<h_{2}<\cdots<h_{m}$ where $a_{g_{i}}$ and $b_{h_{j}}$ are nonzero homogeneous elements of $A$. Since $a \notin Q$, we may assume that $a_{g_{i}} \notin Q$ for all $i$. Now, $d$ being in $\operatorname{Rad}(Q)$ implies that $d^{k} \in Q$ for some integer $k \geq 1$. Hence $a\left(b_{h_{1}}+\cdots+b_{h_{m}}\right)^{k}=a(b-d)^{k} \in Q$ since $a b$ and $d^{k}$ are in $Q$. The component of degree $g_{1}+k h_{1}$ is $a_{g_{1}} b_{h_{1}}^{k}$ which must belong to $Q$ since $Q$ is a graded ideal. But then $b_{h_{1}}^{k} \in \operatorname{Gr}(Q) \subseteq \operatorname{Rad}(Q)$ since $a_{g_{1}} \notin Q$ and $Q$ is a $G$-primary ideal. This implies that $b_{h_{1}} \in \operatorname{Rad}(Q)$ which is a contradiction since $b_{h_{1}} \notin \operatorname{Rad}(Q)$. Hence $Q$ is a primary ideal of $A$. Similarly we can prove that a $G$-prime ideal of $A$ is a prime ideal.

Now we show that $\operatorname{Gr}(Q)=\operatorname{Rad}(Q)$. For, let $r \in \operatorname{Rad}(Q)$, then $r^{n} \in Q$ for some integer $n \geq 1$. Write $r=r_{\gamma_{1}}+r_{\gamma_{2}}+\cdots+r_{\gamma_{t}}$ with $\gamma_{1}<\gamma_{2}<\cdots<\gamma_{t}$ where $r_{\gamma_{1}}, r_{\gamma_{2}}, \ldots, r_{\gamma_{t}}$ are nonzero homogeneous elements of $A$. Since $Q$ is a graded ideal, then homogeneous component of $r^{n}$ whose degree is $n \gamma_{1}$ must belong to $Q$, i.e., $r_{\gamma_{1}}^{n} \in Q$, so it follows that $r_{\gamma_{1}} \in \operatorname{Rad}(Q)$. But then $r_{\gamma_{2}}+\cdots+r_{\gamma_{t}} \in \operatorname{Rad}(Q)$ and we may repeat the argument. Consequently, $r_{\gamma_{i}}^{n_{i}} \in Q$ for some integer $n_{i} \geq 1$ for $i=1,2, \ldots, t$. This implies that $r \in G r(Q)$. Thus $\operatorname{Gr}(Q)=\operatorname{Rad}(Q)$.

Now, let $P \in Z S_{A}^{G}(M)$. Then $A n n(x)$ is a $G$-graded $P$-primary ideal for some nonzero $x \in h(M)$. Consequently, $\operatorname{Ann}(x)$ is a primary ideal and $\operatorname{Rad}(Q)=$ 
$\operatorname{Gr}(Q)=P$, a prime ideal of $A$. Thus $A n n(x)$ is a $P$-primary ideal. Hence $P$ is a Zariski-Samuel prime ideal.

We now prove a graded local-global principle of graded module in terms of strong $G$-Krull prime ideals of $A$.

Theorem 3.18. Let $M$ be a G-graded A-module. Then the following are equivalent:

(1) $M=0$.

(2) $M_{P}^{G}=0$, for all $P \in S K_{A}^{G}(M)$.

(3) $M_{Q}^{G}=0$, for all maximal element $Q \in S K_{A}^{G}(M)$.

Proof. $(1) \Rightarrow(2) \Rightarrow(3)$ Clear.

(3) $\Rightarrow(1)$ Suppose $M_{Q}^{G}=0$ for all maximal element $Q \in S K_{A}^{G}(M)$ and $M \neq 0$. Then $h(M) \neq 0$. Choose an element $0 \neq x \in h(M)$ and a minimal $G$-prime ideal $P$ over $A n n(x)$. Then $P \in A s s_{f}^{G}(M) \subseteq S K_{A}^{G}(M)$. Consider the set $X=\left\{L \in S K_{A}^{G}(M)\right.$ : $P \subseteq L\}$, which is clearly a nonempty set since $P \in X$. Let $\left\{L_{i}\right\}$ be a chain in $X$ and $T=\bigcup L_{i}$. Then $T$ is a $G$-prime ideal of $A$. Now, let $I$ be a finitely generated graded ideal of $A$ such that $I \subseteq T$. Then $I \subseteq L_{i}$ for some $i$. This implies that there exists $0 \neq y \in h(M)$ such that $I \subseteq A n n(y) \subseteq L_{i} \subseteq T$ since $L_{i} \in S K_{A}^{G}(M)$. Thus $T$ is a strong $G$-Krull prime ideal with $P \subseteq T$, and so $T$ is an upper bound for the chain. Then $S K_{A}^{G}(M)$ has a maximal element $Q$ such that $P \subseteq Q$. Thus $\frac{y}{1} \in M_{Q}^{G}=0$. Consequently, there exists $a \in h(A) \backslash Q$ such that $a y=0$. This implies that $a \in \operatorname{Ann}(y) \subseteq P \subseteq Q$, a contradiction. Hence $M=0$.

Let $M$ be a $G$-graded $A$-module. An element $a \in h(A)$ is called a graded zero-divisor on $M$ if $a x=0$ for some non-zero $x \in h(M)$. The set of all graded zero-divisors on $M$ is denoted by $Z^{G}(M)$. Let $Z(M)$ denotes the set of all zero divisors on $M$ in non-graded case. Then in general $Z^{G}(M) \neq Z(M)$. For this, consider the following example.

Example 3.19. Let $A=F[x]$, the polynomial ring over a field $F$. Consider $\mathbb{Z}$ graded ring $A=\bigoplus_{n \in \mathbb{Z}} A_{n}$, where $A_{n}=0$ if $n<0$ and $A_{n}=F x^{n}$ if $n \geq 0$. Let $K=F(x)$, the field of fractions of $A$. Consider a trivially $\mathbb{Z}$-graded $A$-module $M=K / A$. Clearly $Z(M)=A$ and $Z^{G}(M)=\bigcup_{n \geq 0} F x^{n}$. Thus $Z^{G}(M) \neq Z(M)$.

Proposition 3.20. Let $M$ be a $G$-graded A-module. Then $Z^{G}(M)=\bigcup_{P \in S K_{A}^{G}(M)} P^{G}$ where $P^{G}$ denotes the set of all homogeneous elements of $P$.

Proof. Let $a \in Z^{G}(M)$. Then $a x=0$ for some nonzero $x \in h(M)$. Let $P$ be a minimal $G$-prime ideal containing $A n n(x)$. Then $P \in A s s_{f}^{G}(M) \subseteq S K_{A}^{G}(M)$ and 
$a \in P^{G}$. Thus $Z^{G}(M) \subseteq \bigcup_{P \in S K_{A}^{G}(M)} P^{G}$. Conversely, let $a \in \bigcup_{P \in S K_{A}^{G}(M)} P^{G}$. Then $a \in P$ for some $P \in S K_{A}^{G}(M)$, and so there exists $0 \neq x \in h(M)$ such that $a x=0$, i.e., $a \in Z^{G}(M)$. Hence $\bigcup_{P \in S K_{A}^{G}(M)} P^{G} \subseteq Z^{G}(M)$.

\section{Graded primary decomposition in a graded Prüfer domain}

In this section, we study a graded primary decomposition of a graded ideal in a $G$-graded Prüfer domain.

The following example shows that Theorem 2.7 may not work in the graded case.

Example 4.1. Let $A=\mathbb{Q}[x]$ be the polynomial ring. Then $A$ is a Prüfer domain of finite character such that $\operatorname{dim}(A)=1$. Consider $\mathbb{Z}_{2}$-grading on $A$ as in Example 3.7. Here $I=\left(x^{4}-1\right)$ is a graded ideal of $A$ which has a unique primary decomposition $I=J_{1} \cap J_{2} \cap J_{3}$ where $J_{1}=(x+1), J_{2}=(x-1)$ and $J_{3}=\left(x^{2}+1\right)$. Clearly $J_{1}$ and $J_{2}$ are primary ideals but not $\mathbb{Z}_{2}$-primary since $x+1$ and $x-1$ are not homogeneous elements of $A$. On the other hand $I=Q_{1} \cap Q_{2}$ is a $\mathbb{Z}_{2}$-graded primary decomposition of $I$ where $Q_{1}=\left(x^{2}+1\right)$ and $Q_{2}=\left(x^{2}-1\right)$. Thus a primary decomposition and a graded primary decomposition of a graded ideal may be different.

We now generalize Theorem 2.7 to the graded case.

Theorem 4.2. Let $A$ be a $G$-graded Prüfer domain of $G$-finite character with gr$\operatorname{dim}(A) \leq 1$. Then every graded ideal of $A$ can be written as an intersection of finitely many G-primary ideals.

Proof. Let $I$ be a graded ideal of $A$. If $I=\{0\}$, then $I$ is $G$-primary since $A$ is a $G$ graded integral domain. Suppose $I \neq\{0\}$. We claim that $I=\left(\bigcap_{m \in M_{a x}(A)} I A_{m}^{G}\right) \cap A$. Clearly $I \subseteq\left(\bigcap_{m \in \operatorname{Max}^{G}(A)} I A_{m}^{G}\right) \bigcap A$. Now, let $a \in\left(\bigcap_{m \in \operatorname{Max}^{G}(A)} I A_{m}^{G}\right) \bigcap A$ be a homogeneous element. Consider $J=(I: a)=\{b \in A: a b \in I\}$. Then by Proposition 2.4, $J$ is a graded ideal of $A$. If $J \neq A$, then we can choose a $G$-maximal ideal $L$ of $A$ such that $J \subseteq L$. Since $a \in I A_{L}^{G}$, then there exists $b \in h(A) \backslash L$ such that $a b \in I$. Consequently, $b \in J \subseteq L$, which is a contradiction since $b \notin L$. Thus $J=A$, and so $a \in I$. Hence $\left(\bigcap_{m \in \operatorname{Max}^{G}(A)} I A_{m}^{G}\right) \bigcap A \subseteq I$. Now since $A$ is of $G$-finite character, so there are finitely many ideals $m_{1}, m_{2}, \ldots, m_{r} \in \operatorname{Max}^{G}(A)$ such that $I \subseteq m_{i}$. But then $I A_{m}^{G}=A_{m}^{G}$ when $m \neq m_{i}$. Hence $I=I A_{m_{1}}^{G} \cap I A_{m_{2}}^{G} \cap \ldots \cap I A_{m_{r}}^{G} \cap A$. Let us write $Q_{i}=I A_{m_{i}}^{G} \cap A$. Then $I=Q_{1} \cap Q_{2} \cap \ldots \cap Q_{r}$. Now it suffices to show that each $Q_{i}$ is a $G$-graded $P_{i}$-primary ideal of $A$.

Let $a, b \in h\left(A_{m_{i}}^{G}\right)$ such that $a b \in L_{i}=\operatorname{Gr}\left(I A_{m_{i}}^{G}\right)$. Then $(a b)^{n} \in I A_{m_{i}}^{G}$ for some integer $n \geq 1$. Since each $A_{m_{i}}^{G}$ is a $G$-graded valuation domain, then either $a \mid b$ or $b \mid a$. If $a \mid b$, then $b^{2 n} \in I A_{m_{i}}^{G}$ and so $b \in L_{i}$. If $b \mid a$, then $a^{2 n} \in I A_{m_{i}}^{G}$ and so 
$a \in L_{i}$. Thus $L_{i}$ is a $G$-prime ideal of $A_{m_{i}}^{G}$, and so $G$-maximal since $\operatorname{gr}$-dim $(A) \leq 1$ implies $\operatorname{gr}-\operatorname{dim}\left(A_{m_{i}}^{G}\right) \leq 1$. Now, let $a \in G r\left(Q_{i}\right)$. Then $a^{n} \in I A_{m_{i}}^{G}$ for some integer $n \geq 1$ (note that $a \in A$ ). Consequently $a \in L_{i}$, and so $a \in P_{i}=L_{i} \cap A$. On the other hand, let $a \in P_{i}$. Then $a \in L_{i}$, and so $a^{n} \in Q_{i}$ for some integer $n \geq 1$. Hence $\operatorname{Gr}\left(Q_{i}\right)=L_{i} \cap A=P_{i}$. Also, let $a, b \in h(A)$ such that $a b \in P_{i}$. Then $a b \in L_{i}$ and each $L_{i}$ is a $G$-prime ideal of $A_{m_{i}}^{G}$. Consequently $a \in P_{i}$ or $b \in P_{i}$, and so each $P_{i}$ is a $G$-maximal ideal of $A$ since $\operatorname{gr}-\operatorname{dim}(A) \leq 1$. Hence by Proposition 2.5, each $Q_{i}$ is a $G$-graded $P_{i}$-primary ideal of $A$.

It is clear from the definition that a $G$-graded Prüfer domain and a Prüfer domain which is graded by $G$ are different. The following theorem shows the closeness between primary decomposition and graded primary decomposition of a graded ideal of a Prüfer domain which is graded by $G$.

Theorem 4.3. Let $A$ be a Prüfer domain of dimension 1 and of finite character which is graded by a finitely generated abelian group $G$. Let $I$ be a graded ideal of $A$, and $I=\bigcap_{i=1}^{r} Q_{i}$ be a primary decomposition. Let $Q_{i}^{\prime}$ be the largest graded ideal of $A$ such that $I \subseteq Q_{i}^{\prime} \subseteq Q_{i}$. Then $Q_{i}^{\prime}$ is $G$-primary and $I=\bigcap_{i=1}^{r} Q_{i}^{\prime}$ is a G-graded primary decomposition of $I$.

Proof. By Theorem 2.7, $I$ has a primary decomposition say $I=Q_{1} \cap Q_{2} \cap \cdots \cap Q_{r}$ where each $Q_{i}$ is a $P_{i}$-primary ideal of $A$. Let $a, b \in h(A)$ such that $a b \in Q_{i}^{\prime} \subseteq Q_{i}$. Suppose $a \notin Q_{i}^{\prime}$. Then $a \notin Q_{i}$ since $a$ is a homogeneous element and $Q_{i}^{\prime}$ is the largest graded ideal contained in $Q_{i}$. This implies that $b^{n} \in Q_{i}$ for some integer $n \geq 1$, and so $b^{n} \in Q_{i}^{\prime}$ since $b^{n}$ is homogeneous. Let $P_{i}^{\prime}$ be the largest graded ideal of $A$ such that $I \subseteq P_{i}^{\prime} \subseteq P_{i}$ for $i=1,2, \ldots, r$. We show that $P_{i}^{\prime}$ is a $G$-prime ideal of $A$, for let $a, b \in h(A)$ such that $a b \in P_{i}^{\prime}$. Then $a b \in P_{i}$, and so $a \in P_{i}$ or $b \in P_{i}$. This implies that $a \in P_{i}^{\prime}$ or $b \in P_{i}^{\prime}$ since $P_{i}^{\prime}$ is the largest graded ideal contained in $P_{i}$. Thus $P_{i}^{\prime}$ is a $G$-prime ideal and it can be seen that $\operatorname{Gr}\left(Q_{i}^{\prime}\right)=P_{i}^{\prime}$. Thus each $Q_{i}^{\prime}$ is a $G$-graded $P_{i}^{\prime}$-primary ideal. Hence $I=Q_{1}^{\prime} \cap Q_{2}^{\prime} \cap \cdots \cap Q_{r}^{\prime}$ is a $G$-graded primary decomposition of $I$.

As an application of Theorem 4.2, we have the following:

Proposition 4.4. Let $A$ be a $G$-graded Prüfer domain of $G$-finite character with $\operatorname{gr-\operatorname {dim}}(A) \leq 1$. Then the number of minimal $G$-prime ideals over a graded ideal I of $A$ is finite.

Proof. Let $I$ be a graded ideal of $A$. Then by Theorem 4.2, $I=Q_{1} \cap Q_{2} \cap \cdots \cap Q_{r}$ where each $Q_{i}$ is $G$-graded $P_{i}$-primary. Let $P$ be a minimal $G$-prime ideal over $I$. 
Then $Q_{j} \subseteq P$ for some $j$. Therefore $P_{j}=G r\left(Q_{j}\right) \subseteq P$ and so $P=P_{j}$ since $P$ is minimal over $I$. Thus the number of minimal $G$-prime ideals over $I$ is finite.

Remark 4.5. Proposition 4.4 is also true without the condition $\operatorname{gr}-\operatorname{dim}(A) \leq 1$, because each $G$-prime ideal of $A$ is contained in a unique $G$-maximal ideal of $A$.

The following theorem gives a class of examples of QGR-ring.

Proposition 4.6. Let $A$ be a G-graded Prüfer domain of $G$-finite character with $\operatorname{gr-\operatorname {dim}}(A) \leq 1$. Then $A$ is a $Q G R$-ring.

Proof. Let $I$ be a nonzero graded ideal of $A$. Then by Theorem 4.2, $I$ has a reduced $G$-graded primary decomposition say $I=Q_{1} \cap Q_{2} \cap \cdots \cap Q_{r}$ where $Q_{i}$ are $G$-graded $P_{i}$-primary ideals of $A$. We need to show that $Q_{i}$ and $Q_{j}$ are co-maximal when $i \neq j$. Contrary suppose $Q_{i}+Q_{j} \neq A$. Then there exists a $G$-maximal ideal $L$ such that $Q_{i}+Q_{j} \subseteq L$. This implies that $G r\left(Q_{i}\right)+\operatorname{Gr}\left(Q_{j}\right) \subseteq G r\left(Q_{i}+Q_{j}\right) \subseteq L$, i.e., $P_{i}+P_{j} \subseteq L$ where $P_{i}=G r\left(Q_{i}\right)$ is $G$-maximal since gr-dimension of $A$ is 0 or 1 . But then $P_{i}=P_{j}$, which is a contradiction since decomposition is reduced. Thus $Q_{i}+Q_{j}=A$, and so $Q_{i} Q_{j}=Q_{i} \cap Q_{j}$ when $i \neq j$. Consequently, $I=Q_{1} \cdot Q_{2} \ldots Q_{r}$. Hence $A$ is a QGR-ring.

Acknowledgement. The authors sincerely thank the referee for carefully reading, useful suggestions and comments to improve the paper.

\section{References}

[1] D. D. Anderson, D. F. Anderson and G. W. Chang, Graded-valuation domains, Comm. Algebra, 45 (2017), 4018-4029.

[2] D. F. Anderson, G. W. Chang and M. Zafrullah, Graded Prüfer domains, Comm. Algebra, 46 (2018), 792-809.

[3] S. Behara and S. D. Kumar, Group graded associated ideals with flat base change of rings and short exact sequences, Proc. Indian Acad. Sci. Math. Sci., 121 (2011), 111-120.

[4] N. Bourbaki, Commutative Algebra, Chapters 1-7, Translated from the French, Reprint of the 1972 edition, Elements of Mathematics (Berlin), SpringerVerlag, Berlin, 1989.

[5] D. A. Cox, The homogeneous coordinate ring of a toric variety, J. Algebraic Geom., 4 (1995), 17-50.

[6] P. Dutton, Prime ideals attached to a module, Quart. J. Math. Oxford Ser. (2), 29(116) (1978), 403-413. 
[7] N. Epstein and J. Shapiro, Strong Krull primes and flat modules, J. Pure Appl. Algebra, 218 (2014), 1712-1729.

[8] L. Fuchs and E. Mosteig, Ideal theory in Prüfer domains - an unconventional approach, J. Algebra, 252 (2002), 411-430.

[9] J. Iroz and D. E. Rush, Associated prime ideals in non-Noetherian rings, Canad. J. Math., 36(2) (1984), 344-360.

[10] H. A. Khashan, Graded rings in which every graded ideal is a product of Grprimary ideals, Int. J. Algebra, 2(13-16) (2008), 779-788.

[11] S. D. Kumar and S. Behara, Uniqueness of graded primary decomposition of modules graded over finitely generated abelian groups, Comm. Algebra, 39(7) (2011), 2607-2614.

[12] M. D. Larsen and P. J. McCarthy, Multiplicative Theory of Ideals, Pure and Applied Mathematics, 43, Academic Press, New York-London, 1971.

[13] C. Năstăsescu and F. Van Oystaeyen, Graded Ring Theory, North-Holland Mathematical Library, 28, North-Holland Publishing Co., Amsterdam-New York, 1982.

[14] M. Perling and S. D. Kumar, Primary decomposition over rings graded by finitely generated abelian groups, J. Algebra, 318 (2007), 553-561.

[15] M. Perling and G. Trautmann, Equivariant primary decomposition and toric sheaves, Manuscripta Math., 132 (2010), 103-143.

[16] M. Refai and K. Al-Zoubi, On graded primary ideals, Turkish J. Math., 28 (2004), 217-229.

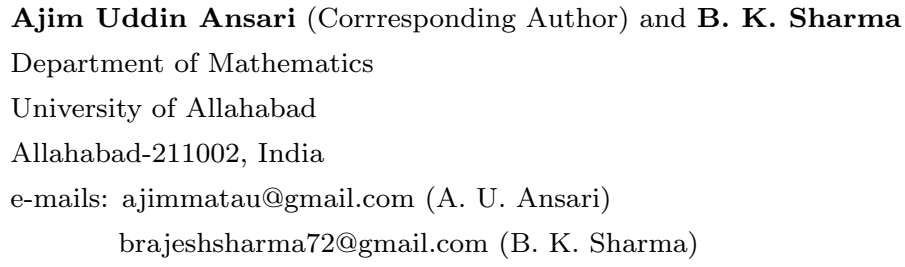

\section{Shiv Datt Kumar}

Department of Mathematics

Motilal Nehru National Institute of Technology

Allahabad-211004, India

e-mail: sdt@mnnit.ac.in 